\title{
ThinPrep Pap Test for Diagnosis of Cervical Cancer in Early Stages
}

\author{
Siamak Sabour ${ }^{1,2}$, Ommolbanin Abbasnezhad ${ }^{1}$, Ali Dastranj Tabrizi ${ }^{3}$, Amir \\ Vahedi $^{4}$, Elmira Mostafidi ${ }^{4}$
}

${ }^{1}$ Department of Epidemiology, School of Public Health, Shahid Beheshti University of Medical Sciences, Tehran, I.R. Iran. ${ }^{2}$ Safety Promotions and Injury Prevention Research Center, Department of Clinical Epidemiology, School of Health, Shahid Beheshti University of Medical Sciences, Tehran, I.R. Iran. ${ }^{3}$ Women's Reproduction Health Research Center, Tabriz University of Medical Science, Tabriz, I.R. Iran. ${ }^{4}$ Department of Pathology, School of Medicine, Tabriz University of Medical Science, Tabriz, I.R. Iran.

\begin{abstract}
Background: Cervical cancer is one of the world's deadliest forms of cancer and Pap smear is the most popular screening test for diagnosis in early stages. Our study aimed to was to assess potential screening rule of ThinPrep Pap test. Methods: In this cross-sectional, study Cervical samples were collected from all women who referred to a pathology center for Papanicolaou test and all samples were prepared with ThinPrep process. To assess validity, biopsy was selected as gold standard. 131 women who had ThinPrep Pap test and biopsy were considered for the analysis. The participants were selected purposefully. Three thresholds were used to define test positivity: 1) Atypical squamous cells of undetermined significance (Asc-us) 2) Low grade squamous intraepithelial lesion (LSIL) 3) High grade squamous intraepithelial lesion (HSIL) and worse. Inter and intra observer reliability were evaluated using kappa (simple and weighted) as well as Fleiss kappa and validity were assessed by the well-known validity estimates for qualitative variables. Results: Intra observer reliability was moderate for pathologists with low and moderate experience (kappa was 0.44 and 0.46 respectively) and was good for experienced pathologist $[$ kappa $(\mathrm{WK})=0.64]$; however, inter observer reliability was poor (Fliess kappa=0.12). For diagnosis of ASCUS and worse, the sensitivity was $96.3 \%$ and for diagnosis of HSIL and worse the sensitivity and specificity were $86.6 \%$ and $95.1 \%$, respectively. Conclusion: ThinPrep pap is an acceptable screening test for diagnosis of cervical cancer in early stages. However, experience and specialty have an effect on reliability's results.
\end{abstract}

Keywords: ThinPrep- Papanicolaou- Validity- Inter and intra observer reliability

Asian Pac J Cancer Care, 4 (4), 141-144

\section{Introduction}

Cancer is one of the main causes of death in the world [1]. Cervical cancer is one of the world's deadliest - but most easily preventable - forms of cancer for women, responsible for more than 270000 deaths annually, $85 \%$ of which occur in developing countries [2]. In Iran, incidence rate of cervical cancer is less than other countries in the world but the risk of mortality of this cancer is high in this region. Also, because cervical cancer is preventable and it can be diagnosed in primary stages, the existence of a screening test is essential [3]. There are several available and affordable tests that can effectively detect pre- cancer, as well as several affordable treatment options [4]. The Pap smear (cytology) is the only test that
Submission Date: 04/15/2019 Acceptance Date: 07/13/2019

has been used in large populations and that has been shown to reduce cervical cancer incidence and mortality [5]. It is generally accepted that pap programs have had significant impact on cervical cancer rates in high resource setting over the past several decades [6].

Liquid-based cytology preparations (LBCP) are replacing conventional Papanicolaou (CP) shown to improve detection rates of cervical intraepithelial neoplasia when compared with conventional preparations. Although the Validity and reliability of a test are one of the most important methodological issues in all research sciences, researchers didn't use the correct statistical methods in many articles [7-11]. More than 170 scientific studies involving the Thin-Prep Pap test have demonstrated its benefits, including increased disease

Corresponding Author:

Dr. Siamak Sabour

Department of Epidemiology, School of Public Health, Shahid Beheshti University of Medical Sciences, Tehran, I.R. Iran.

Email: s.sabour@sbmu.ac.ir 
detection, reduction of equivocal diagnoses, improved specimen adequacy, adjunctive molecular testing and morphology assessment. However, due to lack of studies about reliability and validity of Thin-Prep Pap smear test, doing a study about this important topic seems essential. The aim of our study was to assess potential screening rule of Thin-Prep Pap test to manage patients correctly and diagnose cervical cancer in early stages.

\section{Materials and Methods}

In this cross sectional study, cervical samples were collected from all women who referred to a pathology center for Papanicolaou test and all samples were prepared with Thin-Prep process (CytoFast Company). The women who had a past history of cervical neoplasia and radiation history were excluded from the study. The participants were enrolled between October 2015 and March 2017.

This study investigated reliability and validity of ThinPrep Papanicolaou test. For assessment of inter observer reliability, 97 ThinPrep slides in the pathology center were selected and given to 3 pathologists with different experiences (less experienced, with moderate experience and experienced). We considered pathologist with less than five-year experience as less experienced, five to ten years as moderate and finally more than ten years' experience having fellowship in gynecology pathology as experienced pathologist. Cytology results were reported using the Bethesda system (TBS). Based on this system, pathologists reported the results in 9 categories: 1) Atypical squamous cells of undetermined significance (Asc-us), 2) Atypical squamous cells - cannot exclude HSIL (ASC-H), 3) Low grade squamous intraepithelial lesion (LSIL), 4) High grade squamous intraepithelial lesion (HSIL), 5) cervical intraepithelial neoplasia (CIN1), 6) CIN2,3,7) Squamous cell carcinoma (SCC), 8) Atypical Glandular Cells not otherwise specified (AGC-NOS), 9) Adenocarcinoma. The pathologists reviewed the slides independently and in 3 different centers with a same way. All specimens were taken by two educated and expert health workers so that sampling errors would be low.

For investigation of intra observer reliability, 20 slides from 97 were selected randomly and gave to pathologists after about 4 to 6 weeks so that investigated them once again. In second time observing, the slides number were changed for blinding process.

For investigation of validity, the biopsy was selected as a gold standard. So, all women who had normal and abnormal ThinPrep Pap test and also biopsy were interned to our study. Finally, 131 women who had ThinPrep Pap test and biopsy attended in this study. The biopsy was taken by gynecologists and examination of pathology specimens were carried out by an expert and experienced pathologist. The biopsy results were reported in categories as the same as cytology (pap smear test) results. Therefore the results of histopathology and Pap smear were compared with each other and true and false positive and negative were computed. Three thresholds were used to define test positivity: 1) Atypical squamous cells of undetermined significance (Asc-us) and worse
2) Low grade squamous intraepithelial lesion (LSIL) and worse 3) High grade squamous intraepithelial lesion (HSIL) and worse.

\section{Statistics}

Inter and intra observer reliability was assessed using Fleiss and weighted kappa. For evaluation of validity, the Open Epi website was used. Then validity statistics, sensitivity, specificity, positive predictive value, negative predictive value, positive and negative likelihood ratio, diagnostic accuracy and odds ratio, were calculated for each threshold. We used SPSS version 16.0 for data analysis.

\section{Results}

\section{Intra observer reliability}

In this section, 3 pathologists observed 20 Pap smear slides twice with 4 to 6 weeks interval. The pathologists' reports were considered binary, positive and negative, simple and weighted kappa for every pathologist was calculated. Kappa was 0.46 for the pathologist (with moderate experience), 0.44 for the little experienced pathologist and 0.64 for experienced pathologist.

\section{Inter observer reliability}

Fleiss kappa was 0.11 showing poor inter observer reliability.

\section{Validity analysis}

As for histopathology and thin perp pap smear test's results and using the Open epi web site, sensitivity, specificity, positive and negative predective value, positive and negative likelihood ratio, diagnostic accuracy and odds ratio were calculated for every three threshold. To diagnosis of ASCUS and worse, the sensitivity and specificity were $96.36 \%$ and $34.21 \%$, respectively, for diagnosis of LSIL and worse sensitivity was $86.54 \%$ and specificity was $75.9 \%$ and for diagnosis of HSIL and worse the sensitivity and specificity were $86.67 \%$ and $95.05 \%$, respectively. The results of validity were summarized in Table 1a, b.

\section{Discussion}

The Pap smear is generally accepted as the most successful screening test for cancer detection [12]. We showed that ThinPrep Pap smear is also an acceptable screening test for early detection of cervical cancer. Experienced pathologists in the pathology centers improve the reliability of the ThinPrep in diagnosis of cervical cancers in early stage. Our study will help to gynecologists, midwives and executive managers in public health system to select a correct diagnostic method and manage the patient and costs properly.

In our study, intra observer reliability in pathologists with low and moderate experience was moderate and in experienced pathologist was good. This result indicated that experience influenced on intra observer reliability [13-14]. While in previously published studies, intra 
Table 1a. The Results of Biopsy and ThinPrep Pap Test

\begin{tabular}{lcccccc}
\hline & \multicolumn{2}{c}{ Biopsy } & & \\
& & Normal & ASCUS & ASC-H & LSIL & HSIL \\
\hline Thin-Prep Pap Smear & Normal & 26 & 2 & 0 & 0 & 0 \\
& ASCUS & 27 & 1 & 0 & 4 & 2 \\
& ASC-H & 4 & 0 & 0 & 0 & 1 \\
& LSIL & 15 & 0 & 0 & 0 & 1 \\
& HSIL & 4 & 0 & 0 & 26 \\
\hline
\end{tabular}

Atypical squamous cells of undetermined significance (ASCUS); Low grade squamous intraepithelial lesion (LSIL); High grade squamous intraepithelial lesion (HSIL)

Table 1b. Validity Estimates of the Thin-Prep Pap Smear Test Applying Three Different Cut Off

\begin{tabular}{lccc}
\hline parameter & & Validity estimate & \\
& ASCUS and worse & LSIL and worse & HSIL and worse \\
\hline Sensitivity & $96.3 \%+\mathrm{D} 15: \mathrm{D} 22$ & $86.5 \%$ & $86.6 \%$ \\
Specificity & $34.2 \%$ & $75.9 \%$ & $95.1 \%$ \\
Positive predective value & $51.4 \%$ & $70.3 \%$ & $83.8 \%$ \\
Negative predective value & $92.8 \%$ & $89.5 \%$ & $96 \%$ \\
Positive likelihood ratio & 1.4 & 3.6 & 17.5 \\
Negative likelihood ratio & 0.10 & 0.17 & 0.14 \\
Diagnostic accuracy & $60.3 \%$ & $80.1 \%$ & $93.1 \%$ \\
OR & 13.7 & 20.3 & 124.8 \\
\hline
\end{tabular}

observer reliability was good [15-16]. Inter observer reliability of the Thin-Prep in our study was poor in contrast to the published papers with a moderate reliability [12-17-19].

The reasons that inter and intra observer reliability are poor in our study compared with other studies can be described as below:

1. Lack of enough learning of Thin-Prep Pap smear during residency period. Education period in cytopathology and Pap smear in Iran is lower than in other countries [20-21].

2. Lack of postgraduate training and specialist periodicals for pathologists.

3. The main reason that inters and intra observer reliability is poor, is the lack of specialist pathologists specializing in the field of gynecology. In this study, the pathologists that reviewed slides were general and did not have a specialty in gynecology.

For investigation of validity, 131 women with positive and negative biopsy were interned in this study. Sensitivity for diagnosis of ASCUS and worse was higher than other studies done in the past [22-24]. This is because, in this study, all patients' Pap smear and pathology slides were examined by a pathologist who had fellowship in pathology of gynecology and had high level of experience and skill in this field and all slides were examined with high accuracy. In our study, by increasing the threshold, specificity of the test increased and this was in keeping with other studies [22-24]. These results indicated that Thin-Prep Pap test is a very sensitive to diagnosis of early stages of disease and much more specific in high degree of disease. Also, we showed that the Thin-Prep Pap smear has high sensitivity and has no problems such as sampling errors and low screening quality in compare with conventional Pap smear [24-25]. The sensitivity and other statistics in our study indicated that Thin-Prep Pap test is more valid than the conventional Pap smear.

It is good to know that in many pathology centers screeners examine the pap smear slides to help the pathologists, but there is no supervise and quality control on their performance. This can have negative effect on the correct diagnosis of disease. So, it is recommended that monitor the performance of screeners and pathologists and quality control should be taken into account.

Because the patients' characteristics and clinical data were not available, we could not estimate the added diagnostic value of the Thin-Prep Pap test. We consider this as one of the main limitations of our study.

In conclusion, in summary, based on our results Thin-Prep Pap test can be considered as a screening test for early detection of cervical cancer. However, poor inter observer reliability and moderate intra observer reliability should be taken into account. Experience and specialty affect reliability's results. Therefor using of experienced and specialist pathologists in the pathology centers and quality control on their performance can improve the reliability of the test and help to use this test as a test in diagnosis of cervix cancer in early stages.

\section{Acknowledgements}

This study is a part of MSc thesis supported by Shahid Beheshti University of Medical Sciences.

\section{Funding sources}

This research did not receive any specific grant from 
funding agencies in the public, commercial, or not-forprofit sectors.

\section{Conflict of interest}

The authors declare no conflict of interest.

\section{References}

1. Cervical Cancer 2017. Available from: https://www.cdc.gov/ cancer/cervical/.

2. New WHO guide to prevent and control cervical cancer 2014. Available from: http://www.who.int/mediacentre/news/ releases/2014/preventing-cervical-cancer/en/.

3. Khodakarami N, Farzaneh F, Yavari P, Khayamzadeh M, Taheripanah R, Akbari M. New Cervical Cancer Screening program for Low-risk Iranian Women. The Iranian Journal of Obstetrics, Gynecology And Infertility. 2014;17(95):10.

4. WHO. Comprehensive Cervical Cancer Control, guide to essential practice. second:[Available from: www.who.int/ reproductivehealth.

5. Screening for cervical cancer 2017. Available from: http:// www.who.int/cancer/detection/cervical_cancer_screening/ en/.

6. New evidence on the impact of cervical cancer screening and treatment using HPV DNA tests, visual inspection, or cytology 2009.

7. Sabour S. Validity and reliability of the $13 \mathrm{C}$-methionine breath test for the detection of moderate hyperhomocysteinemia in Mexican adults; statistical issues in validity and reliability analysis. Clin Chem Lab Med. 2014;52(12): e295-6.

8. Sabour S. Validity and reliability of the new Canadian Nutrition Screening Tool in the 'real-world' hospital setting: Methodological issues. Eur J Clin Nutr. 2015;69(7): 864.

9. Sabour S. Adherence to guidelines strongly improves reproducibility of brachial artery flow-mediated dilation. jatherosclerosis. 2016;251: 490.

10. Sabour S. Reliability of a new modified tear breakup time method: methodological and statistical issues. Graefes Arch Clin Exp Ophthalmol. 2016;254(3): 595-6.

11. Sabour S, Abbasnezhad O, Mozaffarian S, Nazari H. Accuracy and Precision in Medical Researches; Common Mistakes and Misinterpretations. World Journal of Research and Review (WJRR). 2017;4(4):58-60.

12. Settakorn J, Rangdaeng S, Preechapornkul N, Nateewatana $\mathrm{S}$, Pongsiralai K, Srisomboon J, et al. Interobserver Reproducibility with LiquiPrepTM Liquid-based Cervical Cytology Screening in a Developing Country. Asian Pacific Journal of Cancer Prevention. 2008; Jan 1;9:92-6.

13. van Grinsven S, Hagenmaier F, van Loon C, van Gorp M, van Kints M, van Kampen A. Does the experience level of the radiologist, assessment in consensus, or the addition of the abduction and external rotation view improve the diagnostic reproducibility and accuracy of MRA of the shoulder? Clinical radiology. 2014;69(11):1157-64.

14. Sabour S. Re: does the experience level of the radiologist, assessment in consensus, or the addition of the abduction and external rotation view improve the diagnostic reproducibility and accuracy of MRA of the shoulder? Clinical radiology. 2015;70(3):333.

15. Klinkhamer P, Vooijs G, Haan A. Intraobserver and interobserver variability in the diagnosis of epithelial abnormalities in cervical smears. Acta Cytol. 1988;32(6): 794-800.

16. Gupta D, Komaromy-Hiller G, Raab S, Nath M. Interobserver and intraobserver variability in the cytologic diagnosis of normal and abnormal metaplastic squamous cells in pap smears. Acta Cytol. 2001;45(5): 697-703.

17. Confortini M, Biggeri A, Cariaggi M, P., Carozz iF, M., Minuti P, A., Russo A, et al. Intralaboratory reproducibility in cervical cytology. Results of the application of a 100-slide set. Acta Cytol. 1992 Dec;37(1):49-54.

18. Sama D, Cotignoli T, Guerrini L, Maioli P, Sintoni C, Bucchi L. Intralaboratory reproducibility of cervical cytology diagnoses in the external quality assurance scheme of the Emilia-Romagna region of Italy. Gynecol Oncol. $1996 \mathrm{Mar}$ 1;60(3): 404-8.

19. Stoler H, Schiffman M. Interobserver Reproducibility of Cervical Cytologic and Histologic Interpretations. Jama. 2001 Mar 21;285(11):1500-5.

20. Educational curriculum of Pathology (in persian). Ministry of Health and Medical Education: 2013.

21. General Pathology. Available from: http://pathology.ubc. $\mathrm{ca} /$ educational-programs/residency-training/generalpathology/.

22. Arbyn M, Sankaranarayanan R, Muwonge R, Keita N, Dolo A, Mbalawa C, G., et al. Pooled analysis of the accuracy of five cervical cancer screening tests assessed in eleven studies in Africa and India. Int J Cancer. $2008 \mathrm{Jul}$ 1;123(1):153-60.

23. Chen C, Yang Z, L iZ, Li L. Accuracy of several cervical screening strategies for early detection of cervical cancer: a meta-analysis. Int J Gynecol Cancer. 2012 Jul 1;22(6):90821

24. Hussein T, Desai M, Tomlinson A, Kitchener H. The comparative diagnostic accuracy of conventional and liquid-based cytology in a colposcopic setting. BJOG: An International Journal of Obstetrics \& Gynaecology. 2005;112(11):1542-6.

25. Farzaneh F, Tamimi M, Amiri Z, Alizadeh K. The value of Pap smear in detecting cervical neoplasia compared with histopathologic findings in patients referred to Taleghani Hospital, Tehran 2007-2009. Pajoohandeh Journal. 2011;16(2):92-7.

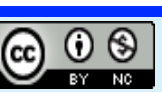

This work is licensed under a Creative Commons AttributionNon Commercial 4.0 International License. 